\title{
Influence of social support on the impact of mobile phone dependence on anxiety levels of Chinese adolescents.
}

\author{
Liu Xiao-Xiao, Dang Jing-Ping*, Tang Yao, Hu Yue-Qi \\ School of Journalism and New Media, Xi'an Jiaotong University, Xi'an, PR China
}

\begin{abstract}
Objective: To investigate the relationship between Mobile Phone Dependence (MPD) of adolescents and their social support-related anxiety levels.

Methods: A total of $\mathbf{1 0 0 6}$ young students selected from Shaanxi Province were examined for their degree of MPD, using Mobile Phone Dependence Index (MPDI), and to obtain their degree of social support and severity of anxiety levels. Social support and anxiety levels were assessed using Social Support Revalued Scale (SSRS) and Self-rating Anxiety Scale (SAS), respectively.

Results: The adolescent's MPAI score of $34.63 \pm 11.53(p<0.05)$ was negatively correlated with social support, but positively correlated with anxiety levels $(\mathbf{p}<0.001)$. Hierarchy regression analysis showed that utilization of social support played a mediating role in the relationship between anxiety and loss of control, and between anxiety and craving $(4.22 \%$ and $4.19 \%$ of the gross effects, respectively).

Conclusion: These results suggest that social support can partially adjust the MPD-induced anxiety levels of adolescents.
\end{abstract}

Keywords: Mobile phone dependence, Anxiety, Social support, Adolescents.

\section{Introduction}

The rapid development of information technology has created "mobile miracle". Mobile phones not only drive social and economic transformation all over the world, but also influence the daily lives of people. The mobile phone use behaviours of adolescents may produce important influences on their physical and psychological health, and may also affect their psychological development. Therefore, the relationship between mobile phone usage behaviours of adolescents and their psychological health deserve research attention.

Studies in China and elsewhere show that university students who overuse mobile phones are more likely to experience anxiety than those who do not. Anxiety has positive correlations with MPD. Indeed, people with MPD have higher anxiety levels than their non-MPD counterparts [1-5]. People with MPD usually avoid and relieve the feeling of anxiety by depending on mobile phones [6]. Social support addresses the effect of stress on people. Stress affects psychological health, especially anxiety. Social support is an important factor for an individual's psychological health. Studies have shown that social support plays a critical mediating role in the relations between MPD and psychological factors of alienation, loneliness and subjective well-being [7-9]. MPD is negatively correlated with social support. A strong social support system is beneficial to the prevention of MPD [7-11]. It has been shown that mobile phone communication does not let users feel better emotional and social support [12]. University students tend to relieve their anxiety through excessive reliance on mobile phones, a practice which unfortunately weakens the social support system, resulting in a vicious circle and aggravating symptoms of MPD and anxiety emotion [13].

Accordingly, the present study was aimed at testing the following hypotheses: (1) MPD can play significant positive prediction role in anxiety; (2) MPD has significant negative correlations with social support; (3) Social support can mediate between MPD and anxiety.

\section{Subjects and Methods}

\section{Study subjects}

The study subjects were students in senior high school and university in Shannxi province, China. A stratified random sampling was used to investigate 16 schools according to district distribution in Shannxi province. Taking each class as a unit, students in junior high school, senior high school and university were selected as study samples according to grade. A total of 1006 undergraduates $(96 \%)$ filled out the questionnaires, but $42(5 \%)$ were excluded from the analysis because they failed to complete the questionnaires in their entirety. Therefore, the final sample consisted of 1048 participants (437 females and 569 males) whose ages ranged from 11 to $26 \mathrm{y}, \mathrm{M}=4.96 \pm 2.10$. Among the 1006 participants were 672 junior high school students, 259 senior high school students and 75 university students. 


\section{Study tools}

The Mobile Phone Addiction Index (MPAI) scale formulated by Leung was used [14]. It has 17 parameters which were applied to diagnose the degree of mobile phone dependence of the adolescent participants (14 to $20 \mathrm{y}$ old). It included four factors: loss of control; anxiety and craving; withdrawal/ escape; and loss of productivity. Likert 5 scale scoring formula was used: the higher the scores, the higher the degree of MPD. Previous research demonstrated that MPAI has strong internal consistency (Cronbach's alpha=0.90) [14]. In the current sample, MPAI scores showed good internal consistency (Cronbach's alpha $=0.881 ; \quad \chi^{2} / \mathrm{df}=3.345, \quad$ RMSEA $=0.068$, $\mathrm{GFI}=0.93$, NFI $=0.907, \mathrm{CFI}=0.932$ ). Ideal indices of fitting were reached which showed good validity of scale.

\section{Self-rating anxiety scale (SAS)}

The SAS is a 20-item self-reported measure of anxiety symptoms, originally developed by Zung in 1971 [15]. Each question was given 4-grade scores according to frequency of corresponding symptoms in the recent one week (scores were given for without/absent, sometimes, often and constant), of which, 5 items were calculated as reverse scores [16]. It has been shown that SAS has good internal consistency (Cronbach's alpha=0.90) [17]. In the current study, Cronbach's alpha was 0.756 . This study gave confirmatory factor analysis for the questionnaire $\left(\chi^{2} / \mathrm{df}=2.416, \mathrm{RMSA}=0.053, \mathrm{CFI}=0.906\right.$, $\mathrm{GFI}=0.944$, NFI $=0.852$, which showed goodness of fit for the factor model of SAS.

\section{Social support re-valued scale (SSRS)}

The SSRS was formulated by Xiao in 1986, and contains 10 items [18]. The SSRS has three dimensions: objective support (three items), subjective support (4 items) and rate of usage of social support (three items). It has moderate internal consistency (Cronbach's alpha coefficients range from 0.69 to 0.83) $[19,20]$. In this study, the Cronbach's alpha for the scale was 0.631 .This study gave confirmatory factor analysis for the questionnaire $\quad\left(\chi^{2} / \mathrm{df}=1.741, \quad \mathrm{P}>0.05, \quad \mathrm{RMSEA}=0.038\right.$, $\mathrm{CFI}=0.981, \mathrm{GFI}=0.989$ and $\mathrm{NFI}=0.957$, all reached to ideal indices of fitting, which indicated good validity of SSRS).

\section{Study procedure and data management}

In early 2016, investigators (head teachers) were given separate training, unified guide and requirements before this investigation. The unit of all investigation was class. Students filled out the questionnaire forms by themselves. The forms were handed out on the spot. Average time for filling out questionnaire was $20 \mathrm{~min}$. Head teachers and students received appreciation for their participation.

\section{Statistical analysis}

EpiData3.1 edition was used to input data. To avoid input error, input records from two independent people were used to proofread input data and raw data. SPSS19.0 software was used for data analysis. Descriptive analysis, Pearson correlation analysis, stepwise regression analysis, and stratified regression analysis were used to do statistical analysis. All analyses were two-sided tests, unless otherwise stated. P value less than 0.05 was considered to be significant.

\section{Results}

\section{Basic features and correlation analysis of MPD, anxiety and social support}

The MPD scores of adolescents with respect to loss of control, anxiety and craving, withdrawal/escape and loss of productivity are shown in Table 1. The MPD scores ranged from 17 to 81 . The total score on mobile dependence was $34.63 \pm 11.53$, while mean score on each MPD item was only $2.04 \pm 0.68$. The total score on loss of control, anxiety and craving, withdrawal/escape, and loss of productivity were $13.74 \pm 4.89,9.46 \pm 4.25,7.34 \pm 3.48$ and $4.09 \pm 2.04$, respectively. These values show the low level of mobile dependence of adolescents in the various dimensions. Mobile dependence of adolescents in this study was at moderate level or below moderate level. Overall, there were no obvious tendencies towards MPD.

The total standard anxiety score of the 1006 adolescents was $46.83 \pm 7.97$. The frequencies of moderate and severe anxiety were $4.8 \%$ and $1.3 \%$, respectively. Total score on social supports among the 1006 adolescents was $33.76 \pm 5.12$. Objective supports scored $9.85 \pm 2.66$, while subjective supports scored $16.45 \pm 2.51$. Score on degree of usage of supports was $7.45 \pm 1.84$.

Correlation analyses were carried out between the various variables of MPD, anxiety and social support, and the results are shown on Table 1. MPD and its four dimensions were positively correlated with anxiety. However, MPD had significant negative correlations with social support, subjective support, and utilization of social support, but had no significant correlations with objective support.

Loss of control had significant negative correlations with social support, subjective support, and utilization of social support. Anxiety and craving had negative correlations with social support, objective support and utilization of social support; while withdrawal/escape had no correlations with social support and the other variables. Loss of productivity had significant correlations with subjective support, and no correlations with social support, objective support and utilization of social support. Anxiety had significant negative 
correlations with social support, subjective support and utilization of social support.

Table 1. Correlation matrix of MPD, anxiety and social support.

\begin{tabular}{|c|c|c|c|c|c|c|c|c|c|c|}
\hline & MPD & M1 & M2 & M3 & M4 & SAS & SSRS & s1 & S2 & S3 \\
\hline MPD & 1 & & & & & & & & & \\
\hline M1 & $0.849^{* \star *}$ & 1 & & & & & & & & \\
\hline M2 & $0.806^{* * *}$ & $0.503^{* *}$ & 1 & & & & & & & \\
\hline M3 & $0.743^{\star \star \star *}$ & $0.447^{\star *}$ & $0.522^{\star \star}$ & 1 & & & & & & \\
\hline M4 & $0.667^{\star \star \star *}$ & $0591^{* *}$ & $0.374^{* *}$ & $0.334^{* * *}$ & 1 & & & & & \\
\hline SAS & $0.259^{* * *}$ & $0.244^{\star \star \star \star}$ & $0.190^{* \star *}$ & $0.168^{\star * *}$ & $0.199^{* * *}$ & 1 & & & & \\
\hline SSRS & $-0.094^{* * *}$ & $-0.139^{* * *}$ & $-0.094^{* *}$ & 0.025 & -0.047 & $-0.105^{* *}$ & 1 & & & \\
\hline S1 & -0.052 & -0.06 & $-0.078^{*}$ & 0.003 & 0.012 & -0.038 & $0.754^{\star * *}$ & 1 & & \\
\hline S2 & $-0.078^{*}$ & $-0.129^{* * *}$ & -0.048 & 0.038 & $-0.094^{* *}$ & $-0.092^{* *}$ & $0.768^{* * *}$ & $0.305^{* * *}$ & 1 & \\
\hline S3 & $-0.082^{* *}$ & $-0.124^{* * *}$ & $-0.082^{* *}$ & 0.014 & -0.021 & $-0.111^{* \star \star x}$ & $0.645^{* \star *}$ & $0.236^{* * *}$ & $0.332^{* \star *}$ & 1 \\
\hline Mean & 34.63 & 13.74 & 9.46 & 7.34 & 4.09 & 46.83 & 33.76 & 9.85 & 16.45 & 7.45 \\
\hline SD & 11.53 & 4.89 & 4.25 & 3.48 & 2.04 & 7.97 & 5.12 & 2.66 & 2.51 & 1.84 \\
\hline
\end{tabular}

Notes: MPD: MPD; M1: Loss of control; M2: Anxiety and craving; M3: Withdrawal/escape; M4: Productivity loss; SAS: Anxiety; SSRS: Social support; S1: Objective support; S2: Subjective support; S3: Utilization of social support; $P^{\star}<0.05, P^{* *}<0.01, P^{* \star *}<0.001$.

\section{Stepwise regression analysis of $M P D$, anxiety and social support}

Based on results in Table 1 and study hypotheses, dimensions which had significant correlations between MPD, various dimensions in social support and anxiety, namely loss of control, anxiety and craving, withdrawal/escape and loss of productivity under MPD; subjective support under social support, and utilization of social support were considered as independent variables and scores on anxiety as dependent variables. The two forms of variables were then given stepwise regression analysis (Table 2). Loss of control, utilization of social support, anxiety and craving, and loss of productivity loss were put into regression analysis. The $\mathrm{F}$ and $\mathrm{T}$ values were significant. The variability of anxiety level was $1.5 \%$. The multiple regression equation was:

\section{$43.623+0.145 \mathrm{X} 1-0.085 \mathrm{X} 2+0.08 \mathrm{X} 3+0.082 \mathrm{X} 4=\mathrm{Y}$}

where $\mathrm{X} 1$ is loss of control; $\mathrm{X} 2$ is utilization of social support; $\mathrm{X} 3$ is anxiety and craving; $\mathrm{X} 4$ is loss of productivity; and $\mathrm{Y}$ is anxiety.

Table 2. Stepwise regression analysis of MPD and social support on anxiety.

\begin{tabular}{|c|c|c|c|c|}
\hline Dependent variable & Sequence of independent variables & $\mathbf{R}^{2}$ & $\beta$ & $\mathbf{t}$ \\
\hline \multirow[t]{4}{*}{ Anxiety } & Loss of control & 0.115 & 0.145 & $3.532^{\star \star *}$ \\
\hline & Utilization of social support & & -0.085 & $-2.78^{* *}$ \\
\hline & Anxiety and craving & & 0.08 & $2.248^{*}$ \\
\hline & Loss of productivity & & 0.082 & $2.165^{*}$ \\
\hline
\end{tabular}

Notes: $\mathrm{P}^{*}<0.05 ; \mathrm{P}^{* *}<0.01 ; \mathrm{P}^{* * *}<0.001$.

\section{Determination of mediation effects of MPD, anxiety and social support}

Based on results on Table 2 from regression analysis and study hypothesis, the variables were put into anxiety regression equation: loss of control, anxiety and craving, withdrawal/ escape and loss of productivity under MPD, and utilization of social support under social support were analyzed for mediation effects. Stratified regression analysis and procedure detection mediation effects were referred to the report of Wen et al. were used to determine mediation effects of the relationship between loss of control, anxiety and craving, loss of productivity, utilization of social support under MPD and anxiety [21]. The results are shown on Table 3. 
First, loss of control was considered as independent variable, anxiety as dependent variable, utilization of social support as mediation variable and these variables were subjected to determination of mediation effect. From Table 2, the regression parameter $\beta$ of each equation was significant $(\mathrm{P}<0.05)$, implying that utilization of social support in the relationship between loss of control and anxiety plays a mediation role, of which the percentage of mediation effect in relation to total effects $((-0.124 \times-0.083) / 0.244)$ was $4.22 \%$.

Second, anxiety-and-craving was considered as independent variable, anxiety as dependent variable, and utilization of social support as mediation variable. They were analyzed for mediation effects. From Table 2, significance detection and regression parameter $\beta$ of each equation were significant $(\mathrm{P}<0.05)$, which shows that utilization of social support in the relationship between anxiety-and-craving, and anxiety plays a mediation function, of which, the percentage of mediation effect in relation to total effects $((-0.082 \times-0.097) / 0.19)$ was $4.19 \%$.

Third, productivity loss was considered as independent variable, anxiety as dependent variable, and utilization of social support as mediation variable. They were analyzed for mediation effects. From Table 3, anxiety and craving, and subjective support parameter $\beta$ in the second step were not significant $(\mathrm{P}>0.05)$. From Sobel detection and SPSS data analysis, it can be seen that $\mathrm{a}=-0.021, \mathrm{~b}=-0.107, \mathrm{Sa}=0.028$ and $\mathrm{Sb}=0.133$. Substituting into the Sobel equation: $\mathrm{Z}$-Value $=\mathrm{a} \times \mathrm{b} /$ SQRT $\left(b^{2} \times \mathrm{Sa}^{2}+\mathrm{a}^{2} \times \mathrm{Sb}^{2}\right)$. The Sobel detection value was 0.549 and $\mathrm{P}=0.583$. This means that utilization of social support had no significant mediation effects between productivity loss and anxiety.

Table 3. Determination of mediation effects of utilization of social support in the relationship between loss of control, anxiety and craving, loss of productivity, and anxiety.

\begin{tabular}{|c|c|c|c|c|c|c|c|c|}
\hline & Step & Dependence Variable & Independence Variable & B & $\mathbf{T}$ & $\mathbf{R}^{2}$ & $\Delta \mathbf{R}^{2}$ & $\mathbf{F}$ \\
\hline \multirow[t]{4}{*}{ Loss of control } & First & Anxiety & Loss of control & 0.244 & $7.964^{* \star *}$ & 0.059 & 0.058 & $63.426^{\star \star \star}$ \\
\hline & Second & Support $\#$ & Loss of control & -0.124 & $-3.958^{\star * \star}$ & 0.015 & 0.014 & $15.664^{\star \star \star}$ \\
\hline & Third & Anxiety & Loss of control & 0.234 & $7.594^{* \star *}$ & 0.066 & 0.064 & $35.508^{\star \star \star}$ \\
\hline & & Usage & Support & -0.083 & $-2.683^{* *}$ & & & \\
\hline \multirow[t]{4}{*}{ Anxiety and craving } & First & Anxiety & Anxiety and craving & 0.19 & $6.132^{* \star *}$ & 0.036 & 0.035 & $37.596^{* * *}$ \\
\hline & Second & Support & Anxiety and craving & -0.082 & $-2.601^{* * *}$ & 0.007 & 0.006 & $6.766^{\star \star *}$ \\
\hline & Third & Anxiety & Anxiety and craving & 0.182 & $5.882^{\star \star \star}$ & 0.045 & 0.043 & $23.826^{\star \star *}$ \\
\hline & & & Support & -0.097 & $-3.119^{\star *}$ & & & \\
\hline \multirow[t]{4}{*}{ Loss of productivity } & First & Anxiety & Loss of productivity & 0.199 & $6.441^{* \star *}$ & 0.04 & 0.039 & $41.49^{* * *}$ \\
\hline & Second & Support & Loss of productivity & -0.021 & -0.654 & 0 & -0.001 & 0.428 \\
\hline & Third & Anxiety & Loss of productivity & 0.197 & $6.404^{* * *}$ & 0.051 & 0.049 & $27.069^{* * *}$ \\
\hline & & & Support & -0.107 & $-3.491^{* *}$ & & & \\
\hline
\end{tabular}

Notes: $\mathrm{P}^{*}<0.05 ; \mathrm{P}^{* *}<0.01 ; \mathrm{P}^{* \star *}<0.001$; \#tilization of social support.

\section{Discussion}

The average score of MPD in this study was $34.63 \pm 11.53$, and. the scores ranged from 17 to 81 , which are lower than the score of $36.7 \pm 11.5$ for MPD of university students investigated by Deng et al. and a value of $36.54 \pm 11.48$ reported in another university investigation by Huang [22,23]. The average score of various features of MPD in this study was only $2.04 \pm 0.68$, and the average scores for loss of control, anxiety and craving, withdrawal/escape, and loss of productivity were all lower than corresponding theoretical scores. These results indicate that the MPD of adolescents in this study was at moderate level or even below moderate. Indeed the MPD tendency was not obvious. Compared with university students, phone use of junior and senior high school students are restricted by schools and family. This will obviously account for low mobile phone tendency amongst adolescents. The total standard score of the 1006 adolescents in this study was $46.83 \pm 7.97$, and the frequency of anxiety was $34.9 \%$. This is in agreement with the total score for anxiety and rate of anxiety of $44.34 \pm 8.32$ and $26.4 \%$, respectively for high school students in Chongqing city [24]. Overall, the degree of anxiety of the 1006 adolescents in this study was higher than average level at home.

The total score for social support of adolescents in this study was $33.76 \pm 5.12$, which is in agreement with the total social support score of $37.62 \pm 16.67$ for high school students in Yunnan province reported by $\mathrm{Li}$ et al., using the same social support evaluation scale [25]. Overall, the social support level of the 1006 adolescents in this study was lower than average level at home. 
MPD is considered an independent variable in mass communication and psychology, while anxiety is taken as dependent variable. In regression analysis, the three dimensions of MPD i.e. loss of control, anxiety-and-craving, and loss of productivity were are all put into the regression equation of anxiety, which showed that MPD can effectively predict the anxiety level of adolescents. This is consistent with the study hypothesis. It shows that uncontrolled mobile phone usage may increase anxiety of adolescents. These results are in agreement with those obtained in earlier studies by Jenaro et al. [1,2,5,26-30]. Adolescents with high mobile dependence level are more likely to feel sad after leaving the mobile phone. If their desire of using mobile phone is not satisfied at that moment, it is likely to produce anxiety.

In this study, scores on social support had significant negative correlations with scores on MPD, loss of control, and anxiety and craving. This is in agreement with the study hypothesis, and it shows that the higher the level of social support, the lower the degree of MPD. The results of stepwise regression analysis showed that utilization of social support could predict anxiety of adolescents. Similar results were reported by Kim et al. [13,31]. From the perspective of media determinism, adolescents have become prisoners of mobile phone. This situation makes them ignore social communication activities in the outside world, which pre-disposes them to individual anxiety.

Results of mediation effect analyses showed that utilization of social support played mediation effects between loss of control and anxiety, and between anxiety-and-craving and anxiety, to the levels of $4.22 \%$ and $4.19 \%$, respectively. However, utilization of social support had no significant correlations with loss of productivity and anxiety, which partly demonstrates the study hypothesis. Adolescents with high degree of MPD are more likely to use mobile phone most of the time. Thus, they tend to spend less time on physical, face-to-face communication, thereby reducing chances of effective material support, family support, and peer support. In this condition, the feeling of anxiety deepens. Adolescents who are addicted to mobile phones are unable to control the length of time spent on mobile phones, which makes them to ignore help and support from others. Since they are unable to receive good support atmosphere form the 'outside world', they experience much more anxiety than non-MPD individuals.

\section{Acknowledgement}

This study was based on National Social Science Fund General Project. It was funded by the National Social Science Foundation of "Influences and strategies of MPD on socialization of adolescents" China (Grant No. 15BXW065). The funders had no role in the study design, data collection and analysis, decision to publish, or preparation of the manuscript.

\section{References}

1. Poorakbaran E. Assessment of using of emerging communication tools (cell phone, internet and satellite) among young adults and its association with anxiety, depression and stress. Fund Mental Health 2015; 17: 254-259.

2. Lee J, Sung MJ, Song SH. Psychological factors associated with smartphone addiction in South Korean adolescents. J Early Adoles 2016.

3. Lou JX, Shen LY, Lin BF. A Report on mobile phone use among college students-a case of Ningbo Institute of Technology, Zhejiang University. Chin Youth Study 2009; 02: $74-80$.

4. Hong FY, Chiu SI, Huang DH. A model of the relationship between psychological characteristics, mobile phone addiction and use of mobile phones by Taiwanese university female students. Comp Hum Behav 2012; 28: 2152-2159.

5. Huang QR, Fan QY, Zeng YF. The relationship between Mobile Surfing Dependency, Mental Health and Personality of College Students. Chin J Health Psychol 2014; 22: 634-636.

6. Long J, Liu TQ, Liao YH. Prevalence and correlates of problematic smartphone use in a large random sample of Chinese undergraduates. BMC Psychiatr 2016; 16: 408.

7. Jiang YZ, Bai XL. On the relationship between college student's mobile phone addiction and loneliness: the intermediary role of online social support. Chin J Special Edu 2015; 1: 41-47.

8. Liu WL, Cai TS. The mediating effect of loneliness between social support and phone addiction tendency. Chin J Clin Psychol 2015; 23: 926-928.

9. Wang YQ, Zhang Y. Relation of mobile phone addiction to perceived social support and subjective well-being in college students. Chin Mental Health J 2015; 29: 868-873.

10. Wei YY. The relationship between social support and mobile phone dependence among college students. J Jilin Business Technol College 2012; 28: 91-93.

11. Ge XH, Zhu ZH. An empirical study on the relationship between adolescent social support and mobile addiction. Chin J Health Statistics 2014; 31: 830-832.

12. Kim JH, Seo M, David P. Alleviating depression only to become problematic mobile phone users: Can face-to-face communication be the antidote? Comp Hum Behav 2015; 51: 440-447.

13. Chen QB, Wu SH. The relationship of college students' dependence on cell phone, social support and anxious feelings. J Wenzhou Vocational Technical College 2016; 16: 82-85.

14. Leung L. Linking psychological attributes to addiction and improper use of the mobile phone among adolescents in Hong Kong. J Children Media 2008; 2: 93-113.

15.Zung WW. A rating instrument for anxiety disorders. Psychosomatics 1971; 12: 371-379. 
16. Han X. A study of undergraduate's mobile phone addition propensity's influence factors and Naikan cognitive therapy on the addition propensity. Soochow University, Suzhou 2014.

17. Long J, Liu TQ, Liao YH. Prevalence and correlates of problematic smartphone use in a large random sample of Chinese undergraduates. BMC Psychiatry 2016; 16: 408.

18. Xiao SY. The theoretical basis and application of $<$ Social Support Rating Scale>. J Clin Psychiatry 1994; 4: 98-100.

19. Liu J, Li F, Lian Y. Investigation of reliability and validity of the social support scale. J Xinjiang Med Univ 2008; 31: 1-3.

20. Sun S, Guan Y, Qin Y. Social support and emotionalbehavioral problems: Resilience as a mediator and moderator. Chin J Clin Psychol 2013; 21: 114-118.

21. Wen ZL, Hou JT, Zhang L. A comparison of moderator and mediator and their applications. Acta Psychologica Sinica 2005; 37: 268-274.

22. Deng ZJ, Huang H, Gui YF. Mobile phone dependence, parenting style and subjective well-being in college students. Chin Mental Health J 2015; 29: 68-73.

23. Huang H, Niu LY, Zhou CY. Reliability and validity of mobile phone addiction index for Chinese college students. Chin J Clin Psychol 2016; 22: 835-838.

24. Wen YT, Li LL, Wang H. Prevalence of anxiety and its related factors among middle school students in Chongqing. Chin J School Health 2010; 31: 776-779.

25. Li XM, Huang Y. Relationship between depression symptoms and social support among high school students in Yunnan province. Chin J Public Health 2015; 31: 1001-1004.
26. Jenaro C, Flores N, Go'Mez-Vela M. Problematic internet and cell-phone use: Psychological, behavioural, and health correlates. Addiction Res Theory 2007; 15: 309-320.

27. Ha JH, Chin B, Park DH. Characteristics of excessive cellular phone use in Korean adolescents. Cyberpsychol Behavior 2008; 11: 783-784.

28. Lu X, Katoh T, Chen Z. Text messaging: Are dependency and excessive use discretely different for Japanese university students? Psychiatry Res 2014; 216: 255-262.

29. İNal EE, Demİrcİ K, Çetİntürk A. Effects of smartphone overuse on hand function, pinch strength, and the median nerve. Muscle Nerve 2015; 52: 183-188.

30. Chen L, Yan Z, Tang W. Mobile phone addiction levels and negative emotions among Chinese young adults: The mediating role of interpersonal problems. Comp Hum Behav 2016; 55: 856-866.

31. Kim JH, Seo M, David P. Alleviating depression only to become problematic mobile phone users: can face-to-face communication be the antidote? Comp Hum Behav 2015; 51: 440-447.

\section{*Correspondence to}

Dang Jing-Ping

School of Journalism and New Media

Xi'an Jiaotong University

Xi'an

PR China 